\title{
Predicting object-mediated gestures from brain activity: an EEG study on gender differences
}

\author{
Vincenzo Catrambone*, Alberto Greco, Giuseppe Averta, Matteo Bianchi, \\ Gaetano Valenza, and Enzo Pasquale Scilingo
}

\begin{abstract}
Recent functional magnetic resonance imaging (fMRI) studies have identified specific neural patterns related to three different categories of movements: intransitive (i.e., meaningful gestures that do not include the use of objects), transitive (i.e., actions involving an object), and tool-mediated (i.e., actions involving a tool to interact with an object). However, fMRI intrinsically limits the exploitation of these results in a real scenario, such as a brain-machine interface (BMI). In this study, we propose a new approach to automatically predict intransitive, transitive, or tool-mediated movements of the upper limb using electroencephalography (EEG) spectra estimated during a motor planning phase. To this end, high-resolution EEG data gathered from 33 healthy subjects were used as input of a three-class k-Nearest Neighbours classifier. Different combinations of EEGderived spatial and frequency information were investigated to find the most accurate feature vector. In addition, we studied gender differences further splitting the dataset into only-male data, and only-female data. A remarkable difference was found between accuracies achieved with male and female data, the latter yielding the best performance $\mathbf{7 8 . 5 5 \%}$ of accuracy for the prediction of intransitive, transitive and tool-mediated actions). These results potentially suggest that different gender-based models should be employed for future BMI applications.
\end{abstract}

\section{INTRODUCTION}

Understanding of how human brain plans and controls movements, especially those related to upper limbs, can be relevant not only from a theoretical point of view, but also for several applications, e.g., in rehabilitation, simplified robot control, or advanced human-robot [1], [2], and brain-machine interfaces [3], through the decoding of the brain activity information.

Upper limb movements are commonly defined in terms of kinematics and dynamics [4]-[7], but they can be also characterised from a neurological point of view as cognitive actions [8], [9]. In the neuroscientific field, the research is mainly oriented towards the detection of possible neural patterns strictly related to different movements, using neuro-imaging techniques: e.g. magneto-electroencephalography [10], [11], fMRI [12], near infra-red spectroscopy [13], and electroencephalography (EEG) [14]-[16]. In past studies, the investigation of motion-related brain areas has been pursued through different kinds of motor tasks that can be grouped into four main categories: motor execution, motor imagery, motor

This research has received funding from the European Union's Horizon 2020 Research and Innovation Programme under Grant Agreement No.688857 (SoftPro).

Authors are with the Department of Information Engineering \& Bioengineering and Robotics Research Center E. Piaggio, School of Engineering, University of Pisa, Pisa, Italy.

* Corresponding author e-mail:

vincenzo. catramboneding.unipi.it observation, and motor planning [17]-[19]. Among these four, motor execution tasks reasonably induce the most noticeable brain response [19], although it represents the most difficult condition to be recorded due to movement artefacts that often affect the measurement, e.g., using fMRI or EEG data. This issue is overcome through protocols that investigate motor imagery, observation, and planning. Indeed, during motor imagery the motion task is only thought or imagined and no movement is actually performed [18], [20]. Protocols investigating motor observation [17], [21], instead, require subjects watching videos of movements, and focus on the action itself, or on the perception provoked by the action [22]. Finally, motor planning [23] is related to the brain activity immediately before the onset of a muscle activation, when the brain is supposed to 'plan' the movement that is going to be performed.

In this study, we focus on EEG signal analysis to investigate human brain activity during upper limb motor-planning tasks. Among the different brain imaging techniques, EEG has a good temporal resolution, but a poor spatial one. Nevertheless, EEG-based studies have already demonstrated the relevance of specific areas and frequency bands during movement tasks. More specifically, parietal and premotor cortices were proven to be involved in both motion execution, imagery, planning, and observation [24]-[26]. Moreover, a decisive role of the central neural cortex during motor execution was identified [14], especially considering oscillations in the $\alpha$ and $\beta$ bands. In addition, an interesting relationship between prefrontal lobe activity and upper limb actions was observed for communicative and behavioural tasks [27]. Other relevant studies have focused on possible brain lateralisation, hypothesising a contralateral control for upper limb movements [28], [29], and an ipsilateral control for hand movements [30], [31]. It is worthwhile noting that many previous studies have highlighted $\alpha, \beta$ and $\gamma$ bands of EEG as most informative (e.g., [32][34]). Indeed $\alpha, \beta$ and $\gamma$ rhythms have been successfully used in a large variety of studies on motor imagery and motor execution [35]-[38], leading to relevant results in psychomotor and BCI applications. Specifically, there are many examples in literature highlighting an $\alpha$ and $\beta$ power suppression or de-synchronization [32], [35], [39]. Furthermore, a $\gamma$ power increase is linked to motor tasks [33], [37].

Of note, while investigating brain dynamics, the gender differences cannot be disregarded [40]. Gender difference is a widely addressed topic in neuroscience, both from an anatomophysiological point of view [41], [42], as well as from a functional and cognitive one [43], [44]. Differences between 
male and female subjects have been found in brain activity related to several tasks that involved cognitive [45], [46], or movement related skills [47], [48] quantified in terms of power activity, symmetry [45], or correlation measures [48]. Other studies have highlighted differences in male/female responses during motor imagery tasks for basic movements [44], [49]. Nevertheless, from the best of our knowledge, this issue has not been addressed neither in motor planning phases nor in actions involving object-related movements.

In this study, we aim at investigating the brain mechanisms underpinning the upper limb movements, also exploited to automatically recognise subject's interaction with objects using EEG information exclusively. This information could be relevant in brain-machine interface (BMI) applications to simplify robot control. For instance, it could be useful to preventively recognise whether an action is object-oriented in order to implement an effective feed-forward motion and manipulation control of a robotic device, e.g. in rehabilitative or assistive robotics. This object-based movement description is based on neuroscientific literature. Indeed, upper limb movements can be grouped in three broad categories according to neuro-psychological and behavioural criteria [8], [50], [51]. More in detail, these three types of movements are: (i) intransitive actions, i.e., not involving the use of any object, yet meaningful and/or communicative; (ii) transitive actions, i.e., characterised by the interaction with a single object; and (iii) tool mediated actions, i.e., indicating tasks where an object is used as a tool to interact with another one [1]. These classes of action have already been extensively described in terms of psychological and behavioural response in several medical studies focused on apraxia [8], [50], [51], i.e., the inability to perform purposive actions as a result of a brain damage. Moreover, the topographical representation of the associated neural activity has been already studied using fMRI [52]. Results have revealed different neural patterns associated with each of these three categories following an experimental protocol based on motor observation. However, fMRI studies have several limitations in terms of versatility, time-resolution and applicability in real BMI scenarios. Here we propose an EEG-based study to devise a pattern recognition system that is able to automatically classify the aforementioned categories of actions, also considering the gender of the subjects.

\section{Materials And Methods}

\section{A. Experimental dataset}

Forty right-handed healthy volunteers provided informed consent to participate in the study. Data from seven subjects were discarded due to artefacts. Therefore, a total of thirty-three subjects was considered for further analyses (17 women, 26.6 years old on average). The experimental protocol comprised 30 right-upper limb movements to be repeated three times (i.e., a grand total of 90 tasks). All experimental procedures were approved by the local ethical committee. As mentioned in the Introduction section, the movements were grouped into three categories, characterised by a different kind of interaction with the object. Specifically, subjects performed:

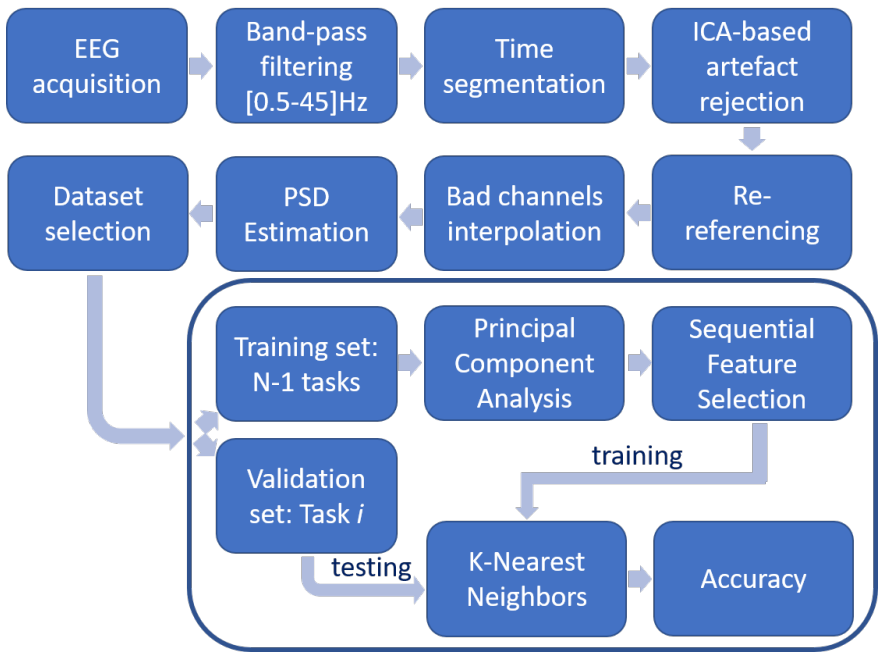

Fig. 1: Block scheme of the data analysis pipeline.

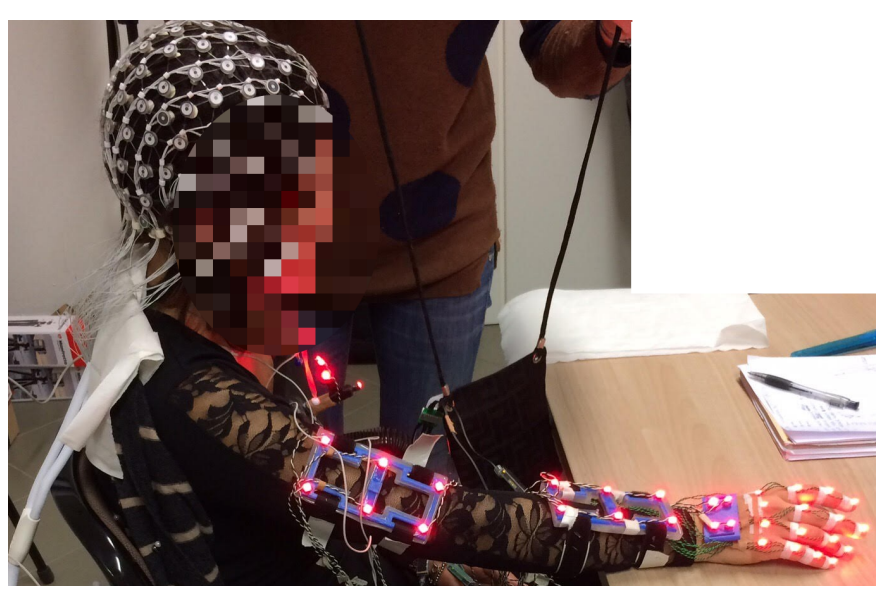

Fig. 2: Exemplary experimental set-up. The subject is equipped with high resolution EEG sensors and active optical markers for motion tracking.

ten intransitive actions, e.g., direct the palm of the right hand in front of the eyes, covering them); ten transitive actions, e.g., reach and grasp a hat, and bring it on the head; and ten tool-mediated actions, e.g., reach and grasp key, use it to open a lock.

Throughout the experiment, brain activity was continuously monitored using a high-density 128 channels Geodesic EEG Systems 300 (Electrical Geodesics, Inc.) with a sampling frequency of $500 \mathrm{~Hz}$. The EEG recording was synchronised with a system for 3D motion tracking using active markers (Phase Space), for kinematic recordings used in other studies. Ten stereo-cameras worked at $480 \mathrm{~Hz}$ tracking 3D position of active markers linked to rigid supports and attached to upper limb links. Exemplary experimental setup is shown in Figure 2 .

At the beginning of each session, the volunteers were instructed on the tasks to perform by an operator mimicking the movements. The actions were mimicked during a short- 


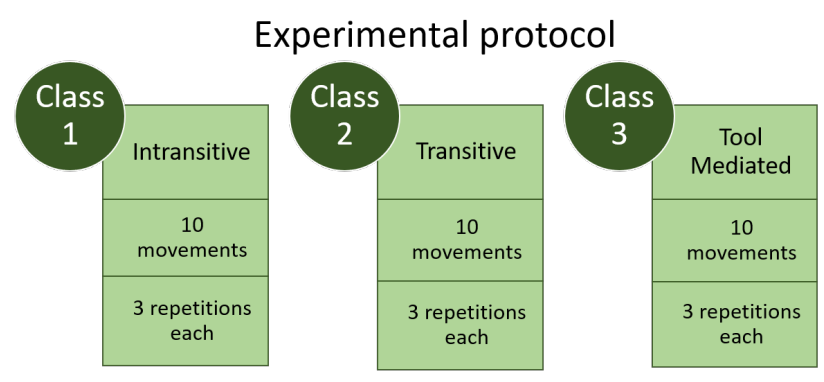

Single trial timeline

Rest $>$ Movement $>$ Rest

Fig. 3: Logic scheme of the experimental protocol timeline comprising three repetitions of ten transitive, intransitive, and tool-mediated movements. Each task included a first resting phase, an actual movement recording, and a final resting state. The highlighted window is the one used in the analysis, considered to be a motor planning phase.

time window before the onset of the first resting phases. Each single task is made of three sequences (see Figure 3): a $3 \mathrm{~s}$ resting state, in which the subjects had to maintain a predefined resting position; the planned actual movement that ended when the subjects returned to the static resting position (the duration of each movement was not constant); additional $3 \mathrm{~s}$ of resting state.

In this study, we considered EEG signals during a $1.5 \mathrm{sec}$ time window that immediately preceded the actual movement execution. This time window can be considered as representing the motor planning brain activity.

\section{B. EEG analysis}

The processing of the EEG signal followed a standard pipeline: bandpass filtering, time segmentation, data rereferencing, corrupted channel rejection, and artefact removal by visual inspection. All the analysis steps were implemented using MatLab software and EEGLAB toolbox [53]. In detail, EEG signals were first filtered through a band-pass Finite Impulse Filter between $0.5 \mathrm{~Hz}$ and $45 \mathrm{~Hz}$ with a Butterworth approximation. Second, EEG segments corresponding to the time window of $1.5 \mathrm{sec}$ that preceded the onset of the actual movement were selected for the motor planning analyses. Third, the corrupted EEG channels were interpolated applying a spherical interpolation algorithm involving the neighbour channels. After that, the EEG signals were re-referenced to the average of all channels, and an Independent Component Analysis decomposition was performed by applying the FastICA algorithm [54] to identify and remove artefacts such as head and arm movements, eye-blinks, heart or muscles electrical activity.

\section{Spectral Analysis}

The power spectral density (PSD) of each EEG electrode was estimated through the spectrogram method. Specifically, we used a non-overlapping Hanning window of 250 samples $(0.5 \mathrm{sec})$, decreasing the variance of the estimation through averaging over subsequent windows. For each action, we computed the PSD within the $1.5 \mathrm{sec}$ anticipating the movement accomplishment, thus limiting our analysis to a motor planning investigation. We computed the PSD in $\alpha[8-12 \mathrm{~Hz}), \beta$ [12-30 Hz) and $\gamma[>30 \mathrm{~Hz}]$ bands, which are commonly considered as the motor-related bands [32]-[34]. Of note, we included also the $\gamma$ band, although it is known to be affected by movement artefacts [55], because we investigate only motor planning tasks.

\section{Data Classifications}

We investigated how gender affects motion classification tasks, and considered three different input datasets:

- the complete dataset, comprised of 2695 observations $(90$ movements $\times 33$ subjects - corrupted discarded tasks).

- the female dataset, comprised of 1301 observations (90 movements $\times 17$ female subjects - corrupted discarded tasks).

- the male dataset, comprised of 1394 observations (90 movements $\times 16$ male subjects - corrupted discarded tasks).

Moreover, for each observation of each input dataset, we considered six different feature-vectors:

- a feature vector of 89 elements where each element represented the PSD computed in the range $[8-45 \mathrm{~Hz}]$, $(\alpha+\beta+\gamma)$

- a feature vector of 89 elements representing the PSD computed in $\alpha$ band;

- a feature vector of 89 elements representing the PSD computed in $\beta$ band;

- a feature vector of 89 elements representing the PSD computed in $\gamma$ band;

- a feature vector of $89 \times 3=267$ elements representing the PSD computed separately in $\alpha, \beta$ and $\gamma$ band;

- a feature vector of 33 elements where each element represented the PSD computed in the range [8-45 Hz], $(\alpha+\beta+\gamma)$ in the central-parietal and prefrontal regions, according to previous findings that indicate them as the brain areas mostly involved in motion activities [14], [24]-[27], [56].

For each combination among the three input datasets and feature vectors, aiming at automatically recognising the three categories of movements (i.e., intransitive, transitive, and toolmediated), we implemented a supervised multi-class pattern recognition system based on K-NN classifier, which is largely used in the literature [57]-[59]. K-NN is a supervised nonparametric classifier that, given a query observation $x_{0}$ and a labelled training set, finds the $\mathrm{K}$ training points $x(r), r=$ $1, \ldots, k$ with the shortest (Euclidean) distance to $x_{0}$, and then classifies the query observation according to the most frequent class among the K-neighbours.

In this study, given the limited number of observations available for the three-class classification task with respect to 
the high number of features and the associated potential risks of overfitting, we applied a principal component (PC) analysis to reduce the dimensionality of each classification problem. The PC analysis was performed on the training set of each fold and the obtained transformation matrix was used on the corresponding validation set. Principal components (PCs) were ranked according to the percentage of their explained variance and were used in a sequential forward selection (SFS) scheme [60]. In conclusion, we propose here a new feature reduction strategy to identify the most informative features as input of the KNN. To assess the out-of-sample predictive accuracy of the system, we adopted a leave one task out (LOTO) crossvalidation procedure.

Following the LOTO procedure, the input feature set was iteratively split into a validation test and a training set. To avoid potential bias, each validation set was comprised of the observations related to the $i$-th movements (i.e., three observation for each subjects), whereas the training set was comprised of the remaining data (see Figure 1). More in detail, each classification task was repeated considering an increasing number of PCs and calculating the accuracy at each iteration using the LOTO cross-validation.

\section{Results}

We show the experimental results in terms of trends of accuracy with respect to the number of selected PCs, also differentiated by gender. First, the results obtained using the single-band feature set are reported. Afterwards, we show the classification results achieved concatenating the three single band PSD to form a unique feature vector. Then, we consider the power spectrum calculated in the whole bandwidth [8-45 $\mathrm{Hz}]$. Finally, results related to the latter approach are shown using only central-parietal subset of electrodes, as mentioned in the previous section. All results are shown considering the three dataset described in section II-D.

\section{A. Single-band power spectrum}

In Figure 4 the balanced accuracy (averaged among positive rates of the three classes), obtained separately from the three considered frequency bands, $\alpha, \beta$ and $\gamma$, respectively is reported. The first row of the figure is related to the accuracy achieved using the complete dataset. In this case a maximum accuracy of $63.38 \%$ was obtained with the first 21 features in the $\beta$ band (less than a fourth of the original feature space dimension). The accuracies with more than 50 PCs are not displayed since they exhibit a decreasing monotone trend. The second and third rows of Figure 4 report on the classification accuracy for male and female sub-groups respectively. In case of male subjects, the accuracy reached up 57\% using 35 features from the $\gamma$ PSD. The female group showed, instead, a much better accuracy of $76.07 \%$, achieved taking only 20 PCs from the $\beta$ PSD. It is worthwhile noting that the classifier trained using female data outperformed the others, and this result is consistent in all the following classifications.

Furthermore, all the accuracy curves present a plateaux with more than 16-18 PCs, demonstrating that the information needed to discriminate among the considered classes (i.e. intransitive, transitive and tool mediated tasks) was almost totally contained in the sub-space made of the first 16-18 PCs. Finally, it can be noticed that $\alpha$ band showed always worse accuracies than $\beta$ and $\gamma$, that, instead, obtained comparable results.

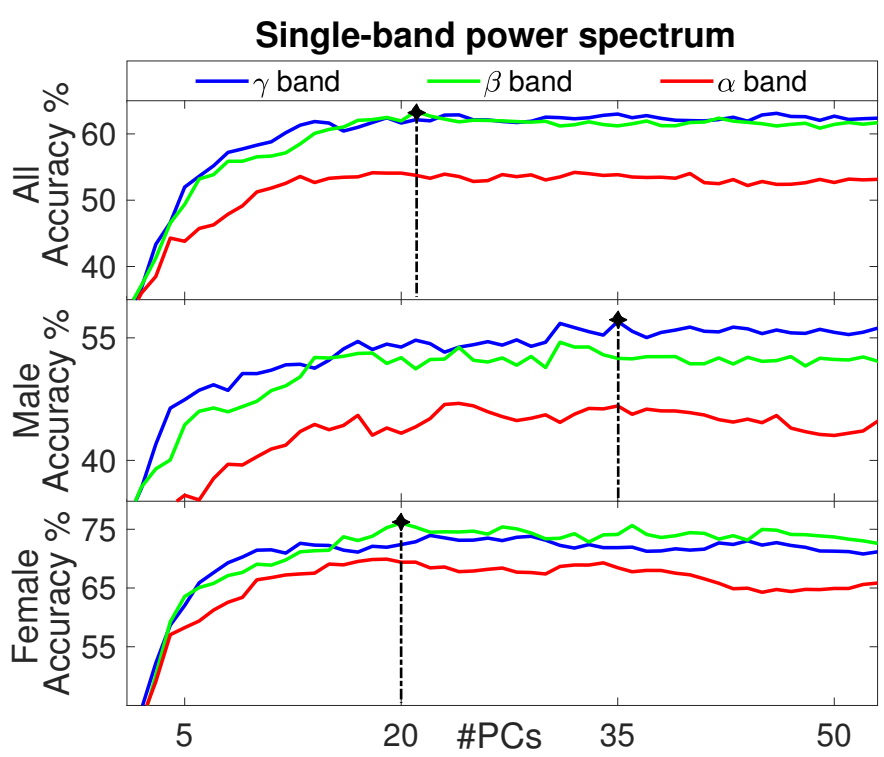

Fig. 4: Classification accuracy w.r.t. the number of principal components (PCs) taken as input features; the dataset involves all the subjects in the first row, only male subjects in the second one, and only female subjects in the third row. The three coloured lines stand for the three frequency bands taken separately as input features, i.e. the red line represents the accuracy achieved using only $\alpha$ band PSD, while blue and green stand for $\gamma$ and $\beta$ respectively. Black dotted lines highlight the points where the maximum is achieved.

\section{B. Concatenated $\alpha, \beta$, and $\gamma$ power spectra}

We considered the feature vector comprising concatenated $\alpha, \beta$, and $\gamma$ PSD from each channel. Red curves in Figure 5 indicate the overall accuracy achieved over the three different classes, while the other curves marked up with the colour blue, green and cyan represent the true positive (TP) rate of the class intransitive, transitive and tool mediated, respectively. Again, the number of PCs was stopped to 50 as the trend over this threshold starts decreasing.

The first row of Figure 5 reports the maximum accuracy of 64.48\% achieved with 24 features for whole dataset analysis. The second and third rows, instead, show the classification results for male and female groups. The highest accuracy (76.05\% using $33 \mathrm{PCs}$ ) was again obtained considering the female subset, while the male dataset with the same number of features reached only a maximum is of $51.64 \%$.

\section{Whole band power spectrum}

Figure 6 shows results of the validation accuracy when the classification procedure is applied to 89 features extracted 
Concatenated $\alpha, \beta$, and $\gamma$ power spectra

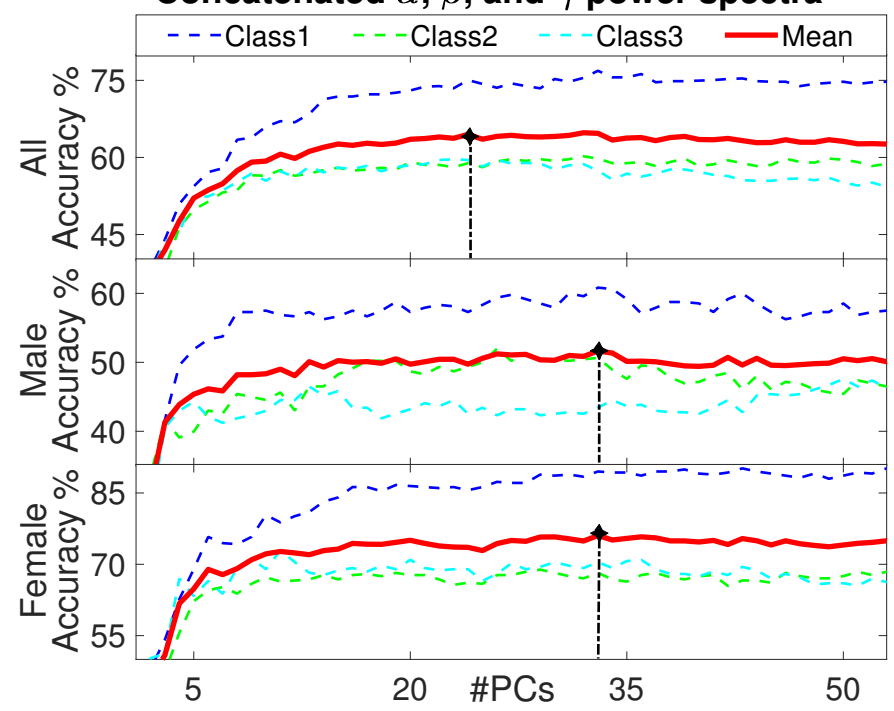

Fig. 5: Classification accuracy vs. the number of principal components (PCs) taken as input features; the dataset involves all the subjects in the first row, only male subjects in the second one and female subjects in the third row. The red thicker curve shows the average accuracy, while the other three coloured lines indicate the three true positive rates, one for each class, i.e. blue curve for intransitive movements, green curve for transitive and cyan for tool mediated. Black dotted lines highlight the points where the maximum is achieved.

calculating the PSD for each electrode in the $[8-45 \mathrm{~Hz}]$ range. Once again the best result was obtained considering the group of women, with a maximum of $78.55 \%$ with 24 PCs. The female set provided also an evident contribute to the $66.35 \%$ of average accuracy reached with the complete dataset. In fact, using only male subjects the maximum was only of $55.34 \%$ with 21 PCs.

\section{Central-parietal-prefrontal subset}

In accordance with previous evidences [14], [24]-[27], [56], we performed a further electrode reduction by selecting only the central, parietal, and prefrontal regions (as shown in Figure 7) and repeating the previous (III-C) analysis. This was applied for a future viable implementation, e.g., in a possible BMI scenario, where physical and eventually economic constraints pose limits on the number of electrodes used for a wearable implementation.

Results are shown in Figure 8: a remarkable gender difference is appreciable also in this case. Indeed, the male dataset achieved the $58.41 \%$ of average accuracy (with 32 PCs), female dataset reached the $76.7 \%$ of validation accuracy, using the first 23 PCs. Concerning the whole dataset, the maximum result of such classification was $66.2 \%$, with 30 PCs.

\section{Discussion AND CONCLUSION}

In this study, we proposed on a novel EEG-based classification analysis to automatically recognise different upper limb

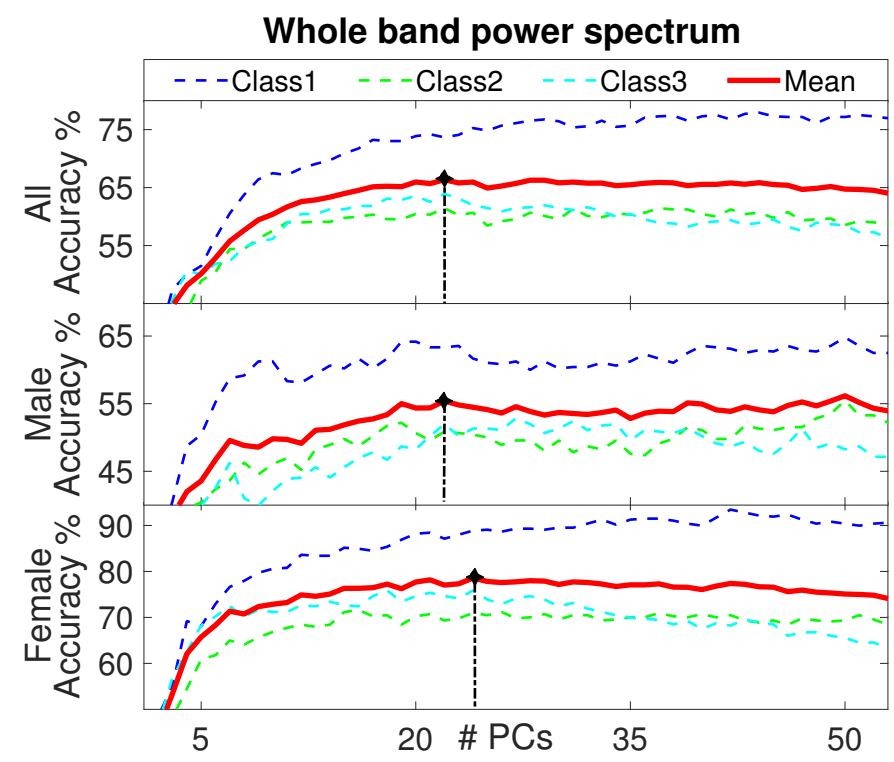

Fig. 6: Accuracy percentages achieved, from the bottom to the top: with only female dataset, only male and both of them. Features were extracted averaging the PSD over the three band frequencies for each channel. Black dotted lines highlight the points where the maximum is achieved.

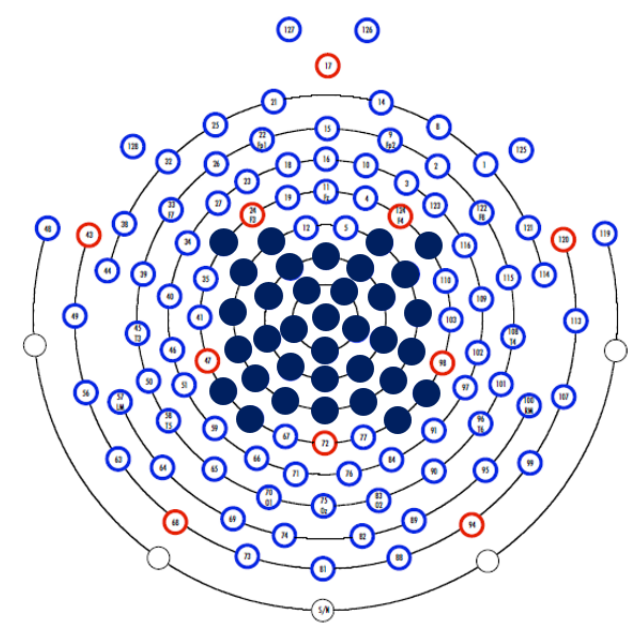

Fig. 7: Here it is showed the high-density 128 -channel cuff used in the experimental recordings. The marked electrodes are the 33 selected as subset of interest in our study.

movements. To this end, EEG signals were gathered from thirty-three healthy right-handed young volunteers, 17 females and 16 males during a motor planning paradigm. Subjects were asked to perform 30 actions belonging to three motion categories, i.e. intransitive, transitive and tool-mediated, as described by [52]. Previous fMRI studies found different topographical representations of the brain activity related to each of these three classes [52]. However, the limitations related to fMRI makes this information difficult to be applied in real scenarios and, from the best of our knowledge, no evidence on EEG data was under this regard. To pave the 


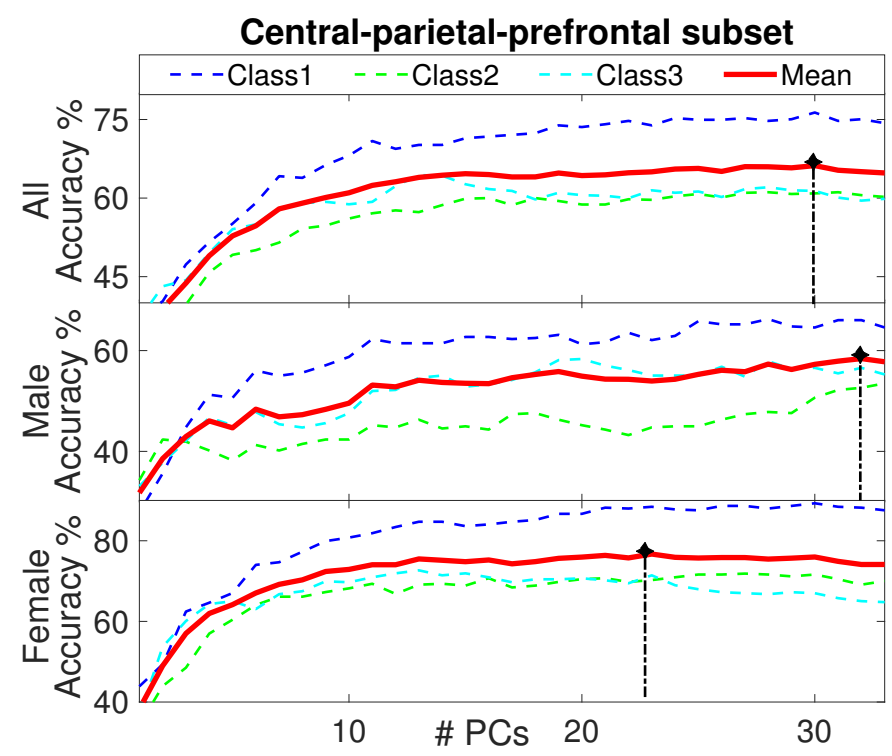

Fig. 8: Accuracy obtained from the classification of the three classes of movement when the subset of electrodes from central-parietal-prefrontal regions is used. The best result in each graph is pointed out by the black dotted line.

path towards future wearable and directly accessible usability for BMI and robotics applications of there results, we thus analysed EEG signals to classify intransitive, transitive and tool mediated actions.

Our analysis focused on the planning phase that precedes the motor action. In this way, not only were we able to investigate a fundamental phase of the motion-related brain activity [19], [23], [61] without movement artefacts, but also to extract a decoded information before the beginning of the action, which can be used to optimise the control strategy for robotic devices in real applications. For example, preventively recognising whether an action is object-based can be used to implement a feedforward control action, eventually complemented with a feedback strategy for motion and manipulative tasks to be performed with robotic manipulators.

A first important aspect of our research was aimed at optimising the classification performance in terms of accuracy, as well as computational cost. Therefore, we applied a new dimensionality reduction and feature selection strategy based on PCA and a SFS scheme. In addition, we proposed also a further analysis in which we performed a prior reduction of EEG channels restricting them to central, parietal, and prefrontal areas of both hemispheres, according to most of the previous results related to motor applications [14], [24][27], [56]. This reduction will likely be a key element for the development of real-application systems, where the quest for wearability limits the number of cumbersome electrodes to be employed for BMI or monitoring of brain activity during the rehabilitative process.

One of the most remarkable findings of our study is the difference in the classification accuracy reachable using only- female and only-male datasets. This result is in agreement with previous motor imagery studies [40], [49], which suggested to take into account the gender when designing and realising BMI systems. Indeed, several studies showed that brain activity in males and females is different when performing cognitive task [45], [46], or movement-related activities [44], [47], [48]. Hence, the female gender is associated with a higher classification accuracy than males, pointing towards a male-specific BCI system. This study has pushed further the investigation in this direction, taking into account for the first time the motion planning phase.

Here, our learning algorithm achieved a good maximum accuracy of $78.55 \%$ in discriminating among intransitive, transitive and tool mediated movements, using only EEG data gathered from female subjects. Besides gender differences, we found that the best result is achieved considering the PSD computed in the whole [8-45 Hz] band. Results get worse if the classifier is trained computing the PSD separately in the $\alpha, \beta$ and $\gamma$ frequency bands (see Figures 4 and 5). Notably, as shown in Figures 5, 8 and 6, intransitive movements were always better recognized than the other two classes. It is reasonable to hypothesize that these involve a different neural dynamics with respect to the other two movement classes which involve interaction with objects. In addition, these results are in agreement with [52] and [62], [63]. A slight decrease of the accuracy is obtained also considering only the electrodes from the central, parietal and prefrontal scalp area, although in this latter case the loss of performance is not relevant compared to the advantage in terms of wearability of this solution.

Concerning the computational cost and the need of reducing the number of features in real applications, our findings suggest that it is more convenient to compress the frequency information integrating a single band rather than to reduce the density of electrodes to a 10-20 standard.

In conclusion, we developed a new pattern recognition system able to accurately predict whether an action performed by the upper limb is going to use an object and the type of interaction with the object itself based only on EEG power spectrum information during motion planning phases. Although our results cannot be directly applied to a real ecological scenario due to the limited resolution in time, they constitute the basis for future applications in robotics and brain-machine interface fields, e.g., for prosthetic feedforward control. In fact, we achieved a satisfactory recognition accuracy while reducing the cardinality of the feature set, so reducing the number of sensors needed for this application and the associated computational cost. Concerning the gender issue only female data allow a good accuracy in the discrimination of motion categories, suggesting a customised gender-based development of BMI. Given the importance of a gender difference in brain activity, the gender issue will deserve a more thorough investigation. Accordingly, future endeavours will be directed towards the improvement of the performance achievable with the male dataset and the implementation of a real-time solution. To this end, further studies will include 
possible relevant information from motion-capture kinematic data, as well as functional connectivity and high-order spectral measures.

\section{APPENDIX A LIST OF MOVEMENTS}

Table I lists the 30 actions included in the applied protocol. The first column counts the task number, the second links to the corresponding grasp type (here we refer to [64]), the third specify the class of movement (according to the categorisation introduced in II-A) and, finally, the fourth column reports a brief description of the task.

\section{REFERENCES}

[1] G. Averta et al., "Unvealing the principal modes of human upper limb movements through functional analysis," Frontiers in Robotics and AI, vol. 4, p. 37, 2017.

[2] C. Della Santina et al., "Postural hand synergies during environmental constraint exploitation," Frontiers in neurorobotics, vol. 11, p. 41, 2017.

[3] M. Santello et al., "Are movement disorders and sensorimotor injuries pathologic synergies? when normal multi-joint movement synergies become pathologic," Frontiers in human neuroscience, vol. 8, p. 1050, 2015.

[4] M. Gabiccini et al., "A data-driven kinematic model of the human hand with soft-tissue artifact compensation mechanism for grasp synergy analysis," in Intelligent Robots and Systems (IROS), 2013 IEEE/RSJ International Conference on, pp. 3738-3745, IEEE, 2013.

[5] J. Lenarcic et al., "Simple model of human arm reachable workspace," IEEE transactions on systems, man, and cybernetics, vol. 24, no. 8, pp. 1239-1246, 1994.

[6] G. Rau et al., "Movement biomechanics goes upwards: from the leg to the arm," Journal of biomechanics, vol. 33, no. 10, pp. 1207-1216, 2000.

[7] H. S. Lo et al., "Exoskeleton robots for upper-limb rehabilitation: State of the art and future prospects," Medical Engineering and Physics, vol. 34, no. 3, pp. 261-268, 2012.

[8] A. Bartolo et al., "Cognitive approach to the assessment of limb apraxia," The Clinical Neuropsychologist, vol. 22, no. 1, pp. 27-45, 2008.

[9] L. J. Buxbaum et al., "Critical brain regions for tool-related and imitative actions: a componential analysis," Brain, vol. 137, no. 7, pp. 1971-1985, 2014.

[10] R. Hari, "Action-perception connection and the cortical mu rhythm," Progress in brain research, vol. 159, pp. 253-260, 2006.

[11] R. Salmelin and R. Hari, "Spatiotemporal characteristics of sensorimotor neuromagnetic rhythms related to thumb movement," Neuroscience, vol. 60, no. 2, pp. 537-550, 1994.

[12] M. Lotze et al., "fmri evaluation of somatotopic representation in human primary motor cortex," Neuroimage, vol. 11, no. 5, pp. 473-481, 2000.

[13] I. Miyai et al., "Cortical mapping of gait in humans: a near-infrared spectroscopic topography study," Neuroimage, vol. 14, no. 5, pp. 11861192, 2001.

[14] P. Avanzini et al., "The dynamics of sensorimotor cortical oscillations during the observation of hand movements: an eeg study," PLoS One, vol. 7, no. 5, p. e37534, 2012.

[15] G. Pfurtscheller et al., "Evaluation of event-related desynchronization (erd) preceding and following voluntary self-paced movement," Clinical Neurophysiology, vol. 46, no. 2, pp. 138-146, 1979.

[16] C. Neuper et al., "Erd/ers patterns reflecting sensorimotor activation and deactivation," Progress in brain research, vol. 159, pp. 211-222, 2006.

[17] J. Decety et al., "Mapping motor representations with positron emission tomography," Nature, vol. 371, no. 6498, p. 600, 1994.

[18] M. Jeannerod, "The representing brain: Neural correlates of motor intention and imagery," Behavioral and Brain sciences, vol. 17, no. 2, pp. 187-202, 1994.

[19] T. Hanakawa et al., "Motor planning, imagery, and execution in the distributed motor network: a time-course study with functional mri," Cerebral cortex, vol. 18, no. 12, pp. 2775-2788, 2008.

[20] P. Dechent et al., "Is the human primary motor cortex involved in motor imagery?," Cognitive Brain Research, vol. 19, no. 2, pp. 138-144, 2004.
TABLE I: List of Movements

\begin{tabular}{|c|c|c|c|}
\hline \# & \# Cut & Class & Description \\
\hline 1 & & Int & Ok gesture (lifting hand from the table) \\
\hline 2 & & Int & Thumb down (lifting hand from the table) \\
\hline 3 & & Int & $\begin{array}{l}\text { Exultation (extending the arm up in the air and } \\
\text { keeping it in with closed fist) }\end{array}$ \\
\hline 4 & & Int & $\begin{array}{l}\text { Hitch-hiking (extending the arm along the frontal } \\
\text { plane, laterally, parallel to the floor, with extended } \\
\text { elbow, closed fist, extended thumb) }\end{array}$ \\
\hline 5 & & Int & $\begin{array}{l}\text { Block out sun from own face (with open hand, } \\
\text { touching the face with the palm and covering the } \\
\text { eyes) }\end{array}$ \\
\hline 6 & & Int & Greet (with open hand, moving wrist) (3 times) \\
\hline 7 & & Int & Military salute (with lifted elbow) \\
\hline 8 & & Int & $\begin{array}{l}\text { Stop gesture (extending the arm along the sagittal } \\
\text { plane, parallel to the floor, with extended elbow, } \\
\text { open palm) }\end{array}$ \\
\hline 9 & & Int & $\begin{array}{l}\text { Pointing (with index finger) of something straight } \\
\text { ahead (with outstretched arm) }\end{array}$ \\
\hline 10 & & Int & $\begin{array}{l}\text { Silence gesture (bringing the index finger, with } \\
\text { the remainder of the hand closed, on the lips) }\end{array}$ \\
\hline 11 & 2 & $\mathrm{Tr}$ & $\begin{array}{l}\text { Reach and grasp a small suitcase (placed along } \\
\text { own frontal plane) from the handle, lift it and } \\
\text { place it on the floor (close to own chair, along } \\
\text { own sagittal plane) }\end{array}$ \\
\hline 12 & 3 & $\operatorname{Tr}$ & $\begin{array}{l}\text { Reach and grasp a glass, drink for } 3 \text { seconds and } \\
\text { place it in the initial position }\end{array}$ \\
\hline 13 & 4 & $\mathrm{Tr}$ & $\begin{array}{l}\text { Reach and grasp a phone receiver (placed along } \\
\text { own sagittal plane), carry it to own ear for } 3 \\
\text { seconds and place it in the initial position }\end{array}$ \\
\hline 14 & 6 & $\mathrm{Tr}$ & $\begin{array}{l}\text { Reach and grasp a book (placed overhead on a } \\
\text { shelf), put in on the table and open it (from right } \\
\text { side to left side) }\end{array}$ \\
\hline 15 & 8 & $\mathrm{Tr}$ & $\begin{array}{l}\text { Reach and grasp a small cup from the handle ( } 2 \\
\text { fingers + thumb), drink for } 3 \text { seconds and place } \\
\text { it in the initial position }\end{array}$ \\
\hline 16 & 11 & $\mathrm{Tr}$ & $\begin{array}{l}\text { Reach and grasp an apple, mimic biting and put } \\
\text { it in the initial position }\end{array}$ \\
\hline 17 & 12,13 & $\mathrm{Tr}$ & $\begin{array}{l}\text { Reach and grasp a hat (placed on the right side of } \\
\text { the table) from its top and place it on own head }\end{array}$ \\
\hline 18 & 12 & $\mathrm{Tr}$ & $\begin{array}{l}\text { Reach and grasp a cup from its top, lift it and put } \\
\text { it on the left side of the table }\end{array}$ \\
\hline 19 & 15 & $\mathrm{Tr}$ & $\begin{array}{l}\text { Receive a tray from someone (straight ahead, with } \\
\text { open hand) and put it in the middle of the table }\end{array}$ \\
\hline 20 & 16 & $\operatorname{Tr}$ & $\begin{array}{l}\text { Reach and grasp a key in a lock (vertical axis), } \\
\text { extract it from the lock and put it on the left side } \\
\text { of the table }\end{array}$ \\
\hline 21 & 1 & T-M & $\begin{array}{l}\text { Reach and grasp a bottle, pour water into a glass } \\
\text { and put the bottle in the initial position }\end{array}$ \\
\hline 22 & $2,3,4$ & T-M & $\begin{array}{l}\text { Reach and grasp a tennis racket (placed along } \\
\text { own frontal plane) and play a forehand (the } \\
\text { subject is still seated) }\end{array}$ \\
\hline 23 & 5 & T-M & $\begin{array}{l}\text { Reach and grasp a toothbrush, brush teeth (hori- } \\
\text { zontal axis, one time left-right) and put it inside } \\
\text { a holder (on the right side of the table) }\end{array}$ \\
\hline 24 & 6 & T-M & $\begin{array}{l}\text { Reach and grasp a laptop, open it (without chang- } \\
\text { ing its position) ( } 4 \text { fingers }+ \text { thumb) }\end{array}$ \\
\hline 25 & $7,8,9$ & T-M & $\begin{array}{l}\text { Reach and grasp a pen (placed on the right side } \\
\text { of the table) and draw a vertical line on the table } \\
\text { (from the top to the bottom) }\end{array}$ \\
\hline 26 & 7 & $\mathrm{~T}-\mathrm{M}$ & $\begin{array}{l}\text { Reach and grasp a pencil (placed along own } \\
\text { frontal plane) ( } 3 \text { fingers + thumb) and put it inside } \\
\text { a squared pencil holder (placed on the left side } \\
\text { of the table) }\end{array}$ \\
\hline 27 & 9 & T-M & $\begin{array}{l}\text { Reach and grasp a tea bag in a cup ( } 1 \text { finger }+ \\
\text { thumb), remove it from the cup and place it on } \\
\text { the table on the right side of the table }\end{array}$ \\
\hline 28 & 10 & T-M & $\begin{array}{l}\text { Reach and grasp a doorknob, turn it clockwise } \\
\text { and counter-clockwise and open the door }\end{array}$ \\
\hline 29 & 13 & T-M & $\begin{array}{l}\text { Reach and grasp a tennis ball (with fingertips) } \\
\text { and place it in a basket on the floor (right) }\end{array}$ \\
\hline 30 & 14 & T-M & $\begin{array}{l}\text { Reach and grasp a cap ( } 2 \text { fingers }+ \text { thumb) of a } \\
\text { bottle (held by left hand), unscrew it and place it } \\
\text { overhead on a shelf }\end{array}$ \\
\hline
\end{tabular}


[21] J. Järveläinen et al., "Activation of the human primary motor cortex during observation of tool use," Neuroimage, vol. 23, no. 1, pp. 187192, 2004

[22] L. C. Quandt et al., "Sensitivity of alpha and beta oscillations to sensorimotor characteristics of action: an eeg study of action production and gesture observation," Neuropsychologia, vol. 50, no. 12, pp. 2745$2751,2012$.

[23] P. Morasso and V. Sanguineti, "Self-organizing body schema for motor planning," Journal of Motor Behavior, vol. 27, no. 1, pp. 52-66, 1995.

[24] S. Caspers et al., "Ale meta-analysis of action observation and imitation in the human brain," Neuroimage, vol. 50, no. 3, pp. 1148-1167, 2010.

[25] D. Arnstein et al., " $\mu$-suppression during action observation and execution correlates with bold in dorsal premotor, inferior parietal, and si cortices," Journal of Neuroscience, vol. 31, no. 40, pp. 14243-14249, 2011.

[26] P. Molenberghs et al., "Brain regions with mirror properties: a metaanalysis of 125 human fmri studies," Neuroscience \& Biobehavioral Reviews, vol. 36, no. 1, pp. 341-349, 2012.

[27] G. Rizzolatti et al., "The mirror-neuron system," Annu. Rev. Neurosci., vol. 27, pp. 169-192, 2004.

[28] G. Pfurtscheller et al., "Motor imagery activates primary sensorimotor area in humans," Neuroscience letters, vol. 239, no. 2-3, pp. 65-68, 1997.

[29] L. Gilden et al., "Summated human eeg potentials with voluntary movement," Electroencephalography and clinical Neurophysiology, vol. 20, no. 5, pp. 433-438, 1966.

[30] E. M. Wassermann et al., "Cortical motor representation of the ipsilateral hand and arm," Experimental Brain Research, vol. 100, no. 1, pp. 121132, 1994.

[31] T. Verstynen et al., "Ipsilateral motor cortex activity during unimanual hand movements relates to task complexity," Journal of neurophysiology, vol. 93, no. 3, pp. 1209-1222, 2005.

[32] D. J. McFarland et al., "Mu and beta rhythm topographies during motor imagery and actual movements," Brain topography, vol. 12, no. 3, pp. 177-186, 2000.

[33] F. P. De Lange et al., "Interactions between posterior gamma and frontal alpha/beta oscillations during imagined actions," Frontiers in human neuroscience, vol. 2, p. 7, 2008.

[34] G. Pfurtscheller et al., "Spatiotemporal patterns of beta desynchronization and gamma synchronization in corticographic data during self-paced movement," Clinical neurophysiology, vol. 114, no. 7, pp. 1226-1236, 2003.

[35] P. Ritter et al., "Rolandic alpha and beta eeg rhythms' strengths are inversely related to fmri-bold signal in primary somatosensory and motor cortex," Human brain mapping, vol. 30, no. 4, pp. 1168-1187, 2009.

[36] G. Pfurtscheller et al., "Separability of eeg signals recorded during right and left motor imagery using adaptive autoregressive parameters," IEEE transactions on Rehabilitation Engineering, vol. 6, no. 3, pp. 316-325, 1998.

[37] H. Yuan et al., "Negative covariation between task-related responses in alpha/beta-band activity and bold in human sensorimotor cortex: an eeg and fmri study of motor imagery and movements," Neuroimage, vol. 49, no. 3, pp. 2596-2606, 2010.

[38] P. Sauseng et al., "Spontaneous locally restricted eeg alpha activity determines cortical excitability in the motor cortex," Neuropsychologia, vol. 47, no. 1, pp. 284-288, 2009.

[39] G. Pfurtscheller et al., "Event-related eeg/meg synchronization and desynchronization: basic principles," Clinical neurophysiology, vol. 110, no. 11 , pp. 1842-1857, 1999.

[40] J. Cantillo-Negrete et al., "Time-frequency analysis of eeg signals from healthy subjects allocated by gender for a subject-independent bci-based on motor imagery," in Neural Engineering (NER), 2013 6th International IEEE/EMBS Conference on, pp. 37-40, IEEE, 2013.

[41] K. Skullerud, "Variations in the size of the human brain. influence of age, sex, body length, body mass index, alcoholism, alzheimer changes, and cerebral atherosclerosis.," Acta neurologica Scandinavica. Supplementum, vol. 102, pp. 1-94, 1985.

[42] H. Haug et al., "The significance of morphometric procedures in the investigation of age changes in cytoarchitectonic structures of human brain.," Journal fur Hirnforschung, vol. 25, no. 4, pp. 353-374, 1984.

[43] J. A. Naglieri and J. Rojahn, "Gender differences in planning, attention, simultaneous, and successive (pass) cognitive processes and achievement.," Journal of Educational Psychology, vol. 93, no. 2, p. 430, 2001.
[44] B. Rescher and P. Rappelsberger, "Gender dependent eeg-changes during a mental rotation task," International Journal of Psychophysiology, vol. 33, no. 3, pp. 209-222, 1999.

[45] J. McGlone, "Sex differences in functional brain asymmetry," Cortex, vol. 14, no. 1, pp. 122-128, 1978.

[46] K. P. Cosgrove et al., "Evolving knowledge of sex differences in brain structure, function, and chemistry," Biological psychiatry, vol. 62, no. 8, pp. 847-855, 2007.

[47] M. J. Farah, "The neural basis of mental imagery," Trends in neurosciences, vol. 12, no. 10, pp. 395-399, 1989.

[48] M. Corsi-Cabrera et al., "Effect of spatial ability and sex inter-and intrahemispheric correlation of eeg activity," Clinical Neurophysiology, vol. 102, no. 1, pp. 5-11, 1997.

[49] J. Cantillo-Negrete et al., "An approach to improve the performance of subject-independent bcis-based on motor imagery allocating subjects by gender," Biomedical engineering online, vol. 13, no. 1, p. 158, 2014.

[50] B. Petreska et al., "Apraxia: a review," Progress in brain research, vol. 164, pp. 61-83, 2007.

[51] L. Canzano et al., "The representation of objects in apraxia: From action execution to error awareness," Frontiers in human neuroscience, vol. 10, 2016.

[52] G. Handjaras et al., "A topographical organization for action representation in the human brain," Human brain mapping, vol. 36, no. 10, pp. 3832-3844, 2015.

[53] A. Delorme et al., "Eeglab: an open source toolbox for analysis of single-trial eeg dynamics including independent component analysis," Journal of neuroscience methods, vol. 134, no. 1, pp. 9-21, 2004.

[54] A. Hyvarinen, "Fast and robust fixed-point algorithms for independent component analysis," IEEE transactions on Neural Networks, vol. 10, no. 3, pp. 626-634, 1999.

[55] J. F. Hipp and M. Siegel, "Dissociating neuronal gamma-band activity from cranial and ocular muscle activity in eeg," Frontiers in human neuroscience, vol. 7, p. 338, 2013.

[56] C. Tzagarakis et al., "Beta-band activity during motor planning reflects response uncertainty," Journal of Neuroscience, vol. 30, no. 34, pp. 11270-11277, 2010.

[57] I. Jekova et al., "Assessment and comparison of different methods for heartbeat classification," Medical Engineering and Physics, vol. 30, no. 2, pp. 248-257, 2008.

[58] J. Lu et al., "Microrna expression profiles classify human cancers," nature, vol. 435, no. 7043, p. 834, 2005.

[59] A. Greco et al., "Brain dynamics induced by pleasant/unpleasant tactile stimuli conveyed by different fabrics," IEEE journal of biomedical and health informatics, 2019.

[60] H. Liu and L. Yu, "Toward integrating feature selection algorithms for classification and clustering," IEEE Transactions on knowledge and data engineering, vol. 17, no. 4, pp. 491-502, 2005.

[61] E. Hoshi and J. Tanji, "Distinctions between dorsal and ventral premotor areas: anatomical connectivity and functional properties," Current opinion in neurobiology, vol. 17, no. 2, pp. 234-242, 2007.

[62] V. Catrambone et al., "Eeg processing to discriminate transitiveintransitive motor imagery tasks: Preliminary evidences using support vector machines," in 2018 40th Annual International Conference of the IEEE Engineering in Medicine and Biology Society (EMBC), pp. 231234, IEEE, 2018.

[63] V. Catrambone et al., "Eeg complexity maps to characterise brain dynamics during upper limb motor imagery," in 2018 40th Annual International Conference of the IEEE Engineering in Medicine and Biology Society (EMBC), pp. 3060-3063, IEEE, 2018.

[64] M. R. Cutkosky, "On grasp choice, grasp models, and the design of hands for manufacturing tasks," IEEE Transactions on robotics and automation, vol. 5, no. 3, pp. 269-279, 1989. 